\title{
Consumo voluntario y digestibilidad en ovinos de henos de pasto guinea (Panicum maximum Jacq.)-clitoria (Clitoria ternatea L Dne.) y de pasto rhodes (Chloris gayana Kunth) cv. Callide ${ }^{1,2}$
}

\author{
Bismarck Sandoval ${ }^{3}$, Elide Valencia ${ }^{4}$, Abner A. Rodríguez ${ }^{5}$ y \\ Paul F. Randel 6
}

J. Agric. Univ. P.R. 93(1-2):41-50 (2009)

\section{RESUMEN}

Se realizó un ensayo con el objetivo de comparar el consumo voluntario (CV) y la digestibilidad de materia seca (MS), de proteína bruta (PB), y de fibra detergente neutro (FDN) de heno de pastos guinea-clitoria (HPGC; $33: 67 \%$ ) y heno de pasto rhodes (HPR). Diez carneros criollos adultos con peso promedio de $35 \mathrm{~kg}$ se distribuyeron entre los henos evaluados según un diseño experimental de intercambio de tratamientos. EI CV fue mayor $(\mathrm{P}<0.05)$ en carneros alimentados con HPGC que con HPR (919 vs. $669 \mathrm{~g}$ MS/d). La digestibilidad de la MS (65.19 vs. $55.51 \%)$ y de la PB (78.80 vs. $65.19 \%)$ también fue mayor $(P<0.05)$ en HPGC que en HPR; mientras, no se encontró diferencia significativa $(P>0.05)$ en la digestibilidad de la FDN entre HPGC $(58.3 \%)$ y HPR (53.9\%). La utilización de HPGC mejoró el consumo de MS digerible por $61 \%$ en términos relativos con respecto al HPR (599 vs. $371 \mathrm{~g} / \mathrm{d}$ ). Se concluye que el HPGC representa una alternativa promisoria para la alimentación de rumiantes.

Palabras clave: consumo voluntario, clitoria, heno de pasto rhodes, ovinos

\section{ABSTRACT}

Voluntary intake and digestibility of guineagrass (Panicum maximum, Jacq.)-clitoria (Clitoria ternatea L Dne.) hay and rhodesgrass (Chloris gayana, Kunth) cv. Callide hay fed to sheep

An experiment was conducted to compare voluntary intake and digestibility of dry matter (DM), crude protein (CP) and neutral detergent fiber (NDF) of guineagrass-clitoria mixed hay $(\mathrm{GCH} ; 33: 67 \%)$ and of rhodesgrass

${ }^{1}$ Manuscrito sometido a la Junta Editorial el 5 de octubre de 2007.

${ }^{2}$ Esta investigación se realizó con fondos USDA/CSREES Proyecto T-STAR 105 y del proyecto SP 389.

${ }^{3}$ Ex-estudiante graduado, Departamento de Cultivos y Ciencias Agroambientales, Box 9000, Universidad de Puerto Rico- Mayagüez, Mayagüez, PR 00068.

${ }^{4}$ Catedrático, Departamento de Cultivos y Ciencias Agroambientales, Box 9000, Universidad de Puerto Rico- Mayagüez, Mayagüez, PR 00681. *Autor para correspondencia: Tel.: 787-265-3851, E-mail address: elideval@uprm.edu

${ }^{5}$ Catedrático, Departamento de Industria Pecuaria.

${ }^{6}$ Catedrático, Departamento de Industria Pecuaria. 
hay (RGH). Ten adult creole rams ( $35 \mathrm{~kg}$ mean liveweight) were assigned to the hays under evaluation according to a crossover experimental design. Higher voluntary DM intake $(\mathrm{P}<0.05)$ was observed in rams fed $\mathrm{GCH}$ than in those fed RGH (919 vs. $669 \mathrm{~g} / \mathrm{d})$. Digestibility of DM (65.2 vs. $55.51 \%$ ) and of CP $(78.8$ vs. $65.2 \%)$ were higher $(P<0.05)$ in $\mathrm{GCH}$ than in $\mathrm{RGH}$, whereas NDF digestibility of GCH $(58.3 \%)$ and of RGH $(53.9 \%)$ did not differ $(P>0.05)$. Feeding GCH resulted in a $61 \%$ relative increase in digestible DM intake with respect to RGH (599 vs. $371 \mathrm{~g} / \mathrm{d}$ ). Guineagrass-clitoria mixed hay is a promising alternative for use in the feeding of ruminants.

Key words: voluntary intake, clitoria, rhodesgrass hay, sheep

\section{INTRODUCCIÓN}

En América Latina y el Caribe la producción de rumiantes se ha basado mayormente en el uso de pastos nativos o naturalizados que en su mayoría no satisfacen los requerimientos nutricionales del animal durante todo el año. Esta deficiencia se refleja en pobre desempeño en términos de ganancia de peso, producción de leche y reproducción animal. Las gramíneas tropicales muestran fluctuaciones en el rendimiento entre la época lluviosa y seca; además, su valor nutritivo en términos de proteína bruta $(\mathrm{PB})$ y digestibilidad in vitro de la materia seca (DIVMS) tiende a ser bajo (Minson, 1990; Lascano y Ávila, 1991).

Una alternativa para mejorar la calidad de la dieta de los rumiantes es mediante el uso de estas gramíneas en asociación con leguminosas. Otra posibilidad es el uso de gramíneas mejoradas, como el pasto rhodes (Chloris gayana Kunth cv. Callide). Las leguminosas incrementan la calidad del forraje consumido y actúan indirectamente al proveer nitrógeno a la gramínea acompañante (Lascano, 2002). A pesar de estas ventajas comprobadas de las leguminosas, su uso en el trópico es limitado. Este uso limitado se debe a la falta de persistencia de las leguminosas en asociaciones, particularmente bajo pastoreo. Se precisan nuevas estrategias de alimentación, siendo la utilización de especies de leguminosas o gramíneas mejoradas conservadas en forma de heno una alternativa interesante.

Una de las leguminosas con potencial para uso en el trópico es la clitoria (Clitoria ternatea L. Dne.). Investigación en México señala que la clitoria tiene un crecimiento inicial rápido y es altamente productiva bajo corte (18 t de MS/ha en siete meses) (Bustamante et al., 2002). Además, su valor nutritivo (18 a $23 \%$ PB y $74 \%$ DIVMS) es comparable al de la alfalfa (Medicago sativa L.). En Puerto Rico, Díaz et al. (2005) incorporaron clitoria en áreas establecidas con pasto guinea (Panicum maximum Jacq.) y lograron incrementar los rendimientos de materia seca (MS) y el valor nutritivo de la gramínea. En investigaciones realizadas en Cuba, vacas alimentadas con el pasto rhodes respondieron fa- 
vorablemente en el consumo de MS y la producción de leche (Hernández et al., 1994a; 1994b).

En Puerto Rico no existe información actualizada sobre el aporte nutricional en términos del consumo voluntario (CV) y digestibilidad del heno de pasto guinea en asociación con clitoria (HPGC), ni del heno de pasto rhodes (HPR) solo. Este estudio se diseñó para comparar el CV y la digestibilidad aparente de los principales nutrientes, MS, PB y fibra detergente neutra (FDN) de HPGC y HPR en carneros adultos.

\section{MATERIALES Y MÉTODOS}

La experimentación se realizó en las facilidades del Departamento de Industria Pecuaria en la Finca Laboratorio Alzamora de la Universidad de Puerto Rico, Recinto Universitario de Mayagüez, y consistió de un ensayo de consumo y digestibilidad con ovinos para evaluar HPGC y HPR. Ambos henos fueron de forrajes cortados a los 42 días de crecimiento aproximadamente en la Estación Experimental Agrícola de Fortuna, Juana Díaz, y en una finca comercial localizada en el municipio de Arecibo, respectivamente.

Como unidades experimentales se utilizaron 10 carneros criollos adultos, de $35 \mathrm{~kg}$ peso vivo (PV) promedio, los cuales se distribuyeron aleatoriamente en jaulas metabólicas individuales provistas de comederos y bebederos. Los animales se esquilaron y desparasitaron (Cydectin; $1 \mathrm{ml} / \mathrm{kg}$ de PV), previo al experimento.

Cada uno de los dos períodos experimentales se dividió en un subperíodo de adaptación de nueve días y en otro de recolección de datos experimentales de seis días. Los nueve días de adaptación permitieron a los carneros acostumbrarse a las condiciones de alojamiento y manejo. Durante este período, todos los animales recibieron heno comercial de pasto guinea como dieta basal y agua fresca ad libitum. El heno ofrecido se cortó a un tamaño aproximado de $10 \mathrm{~cm}$ de largo utilizando una picadora de pastos mecánica (Craftsman $6.5 \mathrm{hp})^{7}$. El objetivo de picar el heno fue reducir la selección animal y facilitar el consumo. Posteriormente, se evaluó el CV y la digestibilidad de HPGC y HPR por seis días.

El suministro de los henos picados se realizó a diario (11:00 a.m.) y la oferta se ajustó de tal forma que los animales no rechazaran más del $20 \%$ del total ofrecido en base seca. Se pesaron los carneros en una báscula antes y después de cada período experimental. En cada segundo subperíodo experimental se cuantificó el alimento ofrecido y rechazado

${ }^{7}$ Las marcas registradas sólo se usan para proveer información específica y su uso no constituye garantía por parte de la Estación Experimental Agrícola de la Universidad de Puerto Rico ni endoso sobre otros productos o equipo que no se mencionan. 
y las heces producidas por cada animal, conservándose diariamente una alícuota de $10 \%$ del total. Al finalizar cada período experimental se formaron muestras compuestas del alimento ofrecido y rechazado y de las heces fecales de cada carnero para su análisis químico posterior.

Las muestras de los tres tipos señalados anteriormente se secaron en un horno de aire forzado (MS; $65^{\circ} \mathrm{C} / 72 \mathrm{~h}$ ) y luego se molieron en un molino Wiley pasándose por un cedazo de $1 \mathrm{~mm}$ de porosidad. Se analizaron las muestras para materia inorgánica (MI; incineración a $550^{\circ}$ C/3 h, usando un Muffle Furnace, Fisher Scientific); la materia orgánica $(\mathrm{MO})$ se determinó por diferencia (MO = 100 - MI), según AOAC (1990).

El porcentaje de nitrógeno se determinó por el método micro-Kjeldhal (utilizando el analizador de nitrógeno Kjeltec system 1002) y luego se calculó la concentración de $\mathrm{PB}(\mathrm{N} * 6.25)$. Las concentraciones de FDN y fibra detergente ácida (FDA) se determinaron utilizando el Fiber Analyzer Ankom 200, siguiendo la metodología de Van Soest et al. (1991). El contenido celular se obtuvo por diferencia (CC = 100 - FDN).

La concentración de hemicelulosa se estimó como la diferencia entre FDN y FDA. La digestibilidad aparente de la materia seca (DMS) y demás fracciones (DPB y DFDN) se calculó como sigue:

$$
\mathrm{DMS}=\frac{\begin{array}{c}
\text { materia seca }(\text { nutriente }) \\
\text { consumido }
\end{array}-\begin{array}{c}
\text { materia seca (nutriente) } \\
\text { excretado }
\end{array}}{\text { materia seca (nutriente) consumido }} \times 100
$$

El diseño experimental utilizado fue un "crossover" (dos períodos con intercambio de tratamientos), con cinco repeticiones de cada tratamiento en cada período. Los datos experimentales de CV, DMS, DPB y DFDN se evaluaron a través de un análisis de varianza (ANOVA) utilizando el procedimiento lineal general (PROC GLM) de SAS (2006). El modelo estadístico utilizado en este estudio fue:

$$
\begin{aligned}
& Y_{i j \mathrm{k}}=\mu+\alpha_{i}+\beta_{\mathrm{j}}+\gamma_{\mathrm{k}}+\varepsilon_{\mathrm{ijK}} \\
& Y_{\mathrm{j} \mathrm{k}}=\text { Variables dependientes (CV, DMS, DPB y DFDN) } \\
& \mu=\text { Media general de la población } \\
& \alpha_{\mathrm{i}}=\text { Efecto del carnero }(1,2,3 \ldots 10) \\
& \beta_{\mathrm{j}}=\text { Efecto de los tratamiento (dos henos diferentes) } \\
& \gamma_{\mathrm{k}}=\text { Efecto del período (primero y segundo de } 15 \text { días) } \\
& \varepsilon_{\mathrm{ijk \textrm {k }}}=\text { Error experimental. }
\end{aligned}
$$

\section{RESULTADOS Y DISCUSIÓN}

La composición química de los henos utilizados se muestra en el Cuadro 1. La concentración de PB (14.3\%) en HPGC (mezcla de gramí- 
CUADRO 1.-Composición química del heno de pasto guinea en asociación con clitoria (HPGC) y del heno de pasto rhodes (HPR) utilizados.

\begin{tabular}{lrr}
\hline Componente $(\%)^{1}$ & HPR & HPGC \\
\hline Humedad & 10.07 & 14.13 \\
Materia seca & 89.93 & 85.87 \\
Materia orgánica $^{2}$ & 91.15 & 90.35 \\
Materia inorgánica $^{2}$ & 8.85 & 9.65 \\
Proteína bruta $^{2}$ & 7.45 & 14.33 \\
FDN $^{2}$ & 73.56 & 61.62 \\
CC $^{2}$ & 26.44 & 38.38 \\
FDA $^{2}$ & 51.68 & 41.71 \\
Hemicelulosa $^{3}$ & 21.88 & 19.91 \\
\hline
\end{tabular}

${ }^{1}$ Medias de cuatro repeticiones.

${ }^{2}$ Base seca.

${ }^{3}$ Calculado por diferencia (FDN-FDA).

nea y leguminosa) fue lógicamente menor a lo típico para heno de clitoria sola (23\%) (Bustamante, 2004; Villanueva et al., 2004). La proporción apreciable de pasto guinea (33\%) en el HPGC también contribuyó a sus altas concentraciones de FDN (61.61\%), de FDA y de hemicelulosa. Según Bustamante (2004) y Villanueva et al. (2004), la FDN en un heno puro de clitoria oscila entre 33 y $38 \%$. La concentración de PB (7.5\%) en el HPR coincide con valores reportados por Wekesa et al. (2006) y Abdulrazak et al. (2005). Este contenido de PB y también el de FDN coinciden con valores de otros henos de gramíneas tropicales (ej., pasto bermuda; Cynodon dactylon) utilizados en ensayos metabólicos anteriores (Rivera, 2003).

\section{Consumo voluntario}

Los CV promedio de la MS, PB y FDN del HPGC y el HPR se presentan en el Cuadro 2. Se observó mayores ( $\mathrm{P}<0.05)$ CV de MS (919 vs. $669 \mathrm{~g} /$ día) y de $\mathrm{PB}$ (140 vs. $59 \mathrm{~g} /$ día) por parte de los carneros alimentados con HPGC que con HPR, pero no se observó diferencia $(\mathrm{P}>0.05)$ en CV de FDN (542 vs. 474 g/día) entre HPGC y HPR, respectivamente.

El CV de MS diario de ambos forrajes con relación al peso corporal no alcanzó el valor esperado de 3\%. Los carneros alimentados con HPGC y HPR consumieron MS equivalente al $2.78 \%$ y $2.03 \%$ de su peso, siendo significativamente diferentes (error estándar de la media de 0.13). Estas diferencias en CV diario de MS (250 g) y de PB (81 g) a favor del HPGC hacen patente el contrastante valor nutritivo de ambos forrajes. Entre los posibles mecanismos responsables del mayor CV de MS de HPGC está el de mayor velocidad de degradación en el rumen (menor tiempo de retención) que hace disponible nuevamente espacio intraruminal. 
CuAdro 2.-Consumo voluntario promedio de heno de pasto guinea en asociación con clitoria (HPGC) y heno de pasto rhodes (HPR).

\begin{tabular}{lrrr}
\hline Componente $(\mathrm{g} / \text { día })^{1}$ & HPR & HPGC & $\mathrm{EE}^{2}$ \\
\hline Alimento ofrecido & 894 & 1148 & 67.91 \\
Materia seca & 69 & 159 & 6.12 \\
Proteína bruta & 652 & 715 & 43.25 \\
Fibra detergente neutro & & & \\
Alimento rechazado & 222 & 231 & 17.81 \\
Materia seca & 13 & 19 & 1.32 \\
Proteína bruta & 181 & 171 & 13.13 \\
Fibra detergente neutro & & & \\
Alimento consumido & & & \\
Materia seca & 669 & 919 & 32.47 \\
Proteína bruta & 58 & 140 & 5.43 \\
Fibra detergente neutro & 474 & 542 & 30.67 \\
\hline
\end{tabular}

${ }^{1}$ Datos en base seca.

${ }^{2} \mathbf{E E}=$ error estándar de la media.

Medias con diferente letra en la misma fila difieren significativamente $(\mathrm{P}<0.05)$.

En estudios metabólicos bajo condiciones similares, Rivera (2003) y Díaz (2004) evaluaron el heno de maní rizomatoso puro (HMR;Arachis glabatra Benth) en términos de CV de MS, PB y FDN en carneros adultos. En los dos estudios el CV de MS del HMR fue 1,000 y $1,005 \mathrm{~g} / \mathrm{d}$, respectivamente. Estos resultados son compatibles con el CV de MS del HPGC encontrado en el presente estudio ( $919 \mathrm{~g} / \mathrm{d})$. Rodríguez et al. (2005) compararon HMR contra heno de alfalfa (Medicago sativa L.) en cuanto a CV en carneros, observando respectivos valores estadísticamente similares (927 y 1,003 g de MS/d) y que concuerdan con el CV presente de HPGC ( $919 \mathrm{~g}$ de MS/d).

Referente al heno de gramínea sola, el CV del HPR (669 g de MS/d) en el presente estudio fue levemente menor a lo reportado por Rivera (2003) para heno de pasto bermuda (HPB; Cynodon dactylon) $(740 \mathrm{~g} / \mathrm{d})$, pero mayor a lo encontrado por Díaz (2004) para heno de pasto pangola (Digitaria eriantha)-guinea en proporciones de 80:20 (473 g/d).

El CV de PB de HPGC ( $140 \mathrm{~g} / \mathrm{d})$ concuerda con valores reportados por Díaz (2004) y Rodríguez et al. (2005) de 149.3 y $144 \mathrm{~g} / \mathrm{d}$, respectivamente. Sin embargo, en este criterio la alfalfa (202 $\mathrm{g} / \mathrm{d}$ ) fue superior al HPGC. Por otro lado, el CV de PB para el HPR (58 g/d) fue superior a lo informado por Díaz (2004) para heno de mezcla de pasto pangola-guinea (23.7 g/d). El CV de FDN del HPGC en el presente estudio (542 g/d) está cercano a los valores encontrados para HMR (Rivera, 2003; Rodríguez et al., 2005), pero mayor que el valor obtenido con heno de alfalfa ( $360 \mathrm{~g} / \mathrm{d})$. 
Bustamante et al. (2002) compararon una ración basal de pasto pará [Brachiaria mutica (Forsk.) Stapf] contra dietas con varias proporciones de inclusion de heno de clitoria $(27,58,87$ y $100 \%$ en base seca) en vacas Pardo Suizo lactantes. Se encontró que el CV de MS, PB y FDN aumentó significativamente al incrementarse la inclusión dietética de heno de clitoria. En varios otros estudios el uso de forraje de leguminosas (fresco o conservado) como suplemento a gramíneas en dietas para rumiantes ha incrementado el CV de MS, PB y FDN (Asefa y Tamir, 2006; Archimides et al., 2001; Rodríguez et al., 1998).

El menor CV de HPR versus HPGC observado en el presente experimento pudo deberse mayormente a diferencias en las características físico-químicas entre los dos henos, especialmente en las concentraciones de PB y FDN. El poco CV obtenido con gramíneas tropicales de baja concentración de PB (menor del 7\% en base seca) se debe en buena medida a que éstas no satisfacen los requerimientos de nitrógeno de los microorganismos ruminales, afectando así la fermentación, la tasa de degradación de la celulosa y la velocidad de pasaje de la digesta (Bustamante et al., 2002; Rodríguez et al., 1998; Poppi y McLennan, 1995). El contenido de PB de $7.45 \%$ del HPR (Cuadro 1) pudo ser marginal al respecto. La mayor concentración de pared celular (FDN), con su aporte de ligno-celulosa, puede afectar el CV por medio de la distensión ruminal, la tasa de degradación de la digesta y el tiempo de retención (Lascano, 2002; Humphreys, 1991; Ruiz y Vázquez, 1983).

\section{Digestibilidad}

La DMS, DPB y DFDN de HPGC y de HPR se presentan en el Cuadro 3. Se observó mayor ( $\mathrm{P}<0.05)$ DMS del HPGC $(65.19 \%)$, superando por 9.68 unidades al HPR (55.51\%). Lo mismo ocurrió, pero más acentuadamente, con DPB, en que el valor del HPGC (78.90\%) superó al del HPR por 19.24 unidades porcentuales. Sin embargo, no se encontró diferencia significativa $(\mathrm{P}>0.05)$ en la DFDN entre HPGC (58.3\%) y HPR $(53.9 \%)$.

CUADRO 3.-Digestibilidad aparente de heno de pasto guinea en asociación con clitoria (HPGC) y heno de pasto rhodes (HPR).

\begin{tabular}{llll}
\hline Componente $(\%)$ & HPR & HPGC & EE $^{2}$ \\
\hline Materia seca & $55.51 \mathrm{~b}$ & $65.19 \mathrm{a}$ & 2.01 \\
Proteina bruta & $59.66 \mathrm{~b}$ & $78.80 \mathrm{a}$ & 1.85 \\
Fibra detergente neutro & 53.86 & 58.33 & 1.31 \\
\hline
\end{tabular}

'Base seca.

${ }^{2} \mathbf{E E}=$ error estándar de la media.

Medias con diferente letra en la misma fila difieren significativamente $(\mathrm{P}<0.05)$. 
La DMS del HPGC (65.19\%) fue igual a la de HMR (65.20\%) pero menor a la de heno de alfalfa (70.0\%) determinadas en estudios previos. La DPB del HPGC (78.80\%) fue superior a valores determinados por Rodríguez et al. (2006) para HMR $(69.50 \%)$ y alfalfa (74.50\%).

La DMS para el HPR (55.51\%) obtenida en el presente estudio fue similar a lo encontrado por Díaz (2004) en heno de pasto pangola-guinea (HPG) (53.0\%). La DPB (59.66\%) del HPR también concuerda con los resultados de Díaz (2004) para el HPG (58.0\%), pero difiere a lo encontrado por Rivera (2003) en el HPB (33.40\%).

En otros trabajos relacionados se ha observado mayor DMS, DFDN y DPB al evaluarse crecientes niveles de suplementación $(0,15$ y $30 \%$ en la MS) de heno de alfalfa en dietas para vaquillas basadas en HPB y heno de pata de gallo (Dactylis glomerata) (Lagasee, 1990). En numerosos otros estudios el uso de forraje de leguminosas (fresco o conservado) como suplemento de dietas para rumiantes a base de gramíneas ha incrementado la digestibilidad (Bochelie y Tamir, 2006; Archimides et al., 2001; Lascano y Palacios, 1993).

En este estudio se observó mayor DMS y DPB de HPGC que de HPR. Esta diferencia pudo estar relacionada en parte a una mayor disponibilidad de nitrógeno a nivel ruminal en carneros alimentados con HPGC que con HPR, por medio de una mayor actividad microbiana para degradar el forraje (Bustamante, 2002; Poppi y McLennan, 1995; Humphreys, 1991). Una disponibilidad subóptima de nitrógeno en el rumen atrasa la degradación de los sustratos (Hespell y Bryant, 1979). El HPR presentó mayor concentración de FDN y FDA comparado con el HPGC, pero la DFDN de ambos henos fue similar. Este resultado puede señalar una relativa similitud en las propiedades químicas intrínsecas de los carbohidratos estructurales presentes en la pared celular de estos dos henos.

El presente estudio brinda información sobre el aporte nutricional en términos del CV y la digestibilidad aparente del HPGC y HPR. La utilización de HPGC mejoró el consumo por $27 \%$ y la digestibilidad por $17 \%$ en términos relativos con respecto al HPR, lo cual conduce a un aumento relativo de $61 \%$ en el consumo de MS digerible (599 vs. $371 \mathrm{~g} / \mathrm{d}$ ). Queda demostrado que el HPGC es una alternativa promisoria en la alimentación de pequeños rumiantes. No obstante, es necesario evaluar el efecto de niveles de suplementación utilizando forraje fresco de clitoria en dietas basadas en gramíneas tropicales sobre otros criterios del desempeño animal, como lo son la ganancia de peso y la producción de leche.

\section{CONCLUSIÓN}

El HPGC evaluado fue superior en valor nutritivo al HPR en términos de CV y digestibilidad de los principales nutrientes. El HPR mostró un valor nutritivo semejante a heno de otras gramíneas tropicales comunes. 


\section{LITERATURA CITADA}

Abdulrazak, S. A., G. E. Njuguna y K. P. Karau, 2005. The effect of supplementing rhodes grass (Chloris gayana) hay with Acasia tortilis leaves and pod mixture on intake, digestibility and growth performance of goats. Livestock Research for Rural Development 17(12):1-7.

A.O.A.C. (Association of Official Analytical Chemists), 1990. Official Methods of Analysis, 13th ed. Washington, D. C.

Archimede, H., M. Dulorme, R. Tournebize, G. Saminadin, F. Periacarpin y A. Xande, 2001. The effects of Gliricidia supplementation on intake and digestion of a Digitaria decumbens hay by Black-belly sheep. J. Agric. Sci. Camb. 137(1):105-111.

Asefa, G. y B. Tamir, 2006. Effects of supplementing different forms of Acasia saligna leaves to grass hay on feed intake and growth of lambs. Trop. Sci. 46(4):205-208.

Bochelie, D. y B. Tamir, 2006. Effect of supplementing different level of tagasaste (Chamaecytisus palmensis) to grass hay on nutrient intake and digestibility by lambs. Trop. Sci. 46 (4):189-191.

Bustamante, G. J. J., 2004. Utilización de heno de Clitoria en la alimentación de becerras lactantes de propósito lechero. INIFAP. Folleto Científico No.1. p. 3-30.

Bustamante, G. J. J., V. F. J. Ávalos, B. A. J. Cárdenas y R. V. J. Ceja, 2002. Utilización del heno de clitoria (Clitoria ternatea L.) en la alimentación de vacas pardo suizo en lactación. Revista Técnica Pecuaria México 42(3):477-487.

Díaz, H., J. T. Rousse y E. Valencia, 2005. Seedbed management effects on botanical composition and nutritive value of bluepea (Clitoria ternatea) on a guineagrass (Panicum maximum Jacq.) stand. Proc. Caribbean Food Crops Society 41(2):578.

Díaz, H., 2004. Efecto de la suplementación con ensilaje de residuos de una planta procesadora de tilapia (Oreochromis niloticus) sobre el consumo voluntario y la digestibilidad de nutrientes de heno de gramíneas y leguminosas tropicales. Tesis MS. Universidad de Puerto Rico, Recinto Universitario de Mayagüez.

Hespell, R. B. y M. P. Bryant, 1979. Efficiency of rumen microbial growth: influence of some theoretical and experimental factors on Yatp. J. Animal Sci. 49:1640-1659.

Hernández, D., M. Carballo, C. Mendoza y C. Fung, 1994a. Estudio del manejo de Chloris gayana cv. Callide para la produceión de leche. I. Efecto del tiempo de estancia. Pastos y Forrajes 17:165-172.

Hernández, D., M. Carballo, C. Mendoza y C. Fung, 1994b. Estudio del manejo de Chloris gayana cv. Callide para la producción de leche. II. Efecto de la oferta diaria de materia seca. Pastos y Forrajes 17:245-255.

Humphreys, L. R., 1991. Tropical Pasture Utilization. Cambridge University Press. Cambridge, UK. p. 1-172.

Lagasse, M. P., 1990. Effects of supplemental alfalfa hay on feed intake and digestion by Holstein steers consuming high-quality bermudagrass or orchardgrass hay. J. Animal Sci. 68:2839-2847.

Lascano, C. E., 2002. Caracterización de las pasturas para maximizar producción animal. Archivos Latinoamericanos de Producción Animal 10(2):126-132.

Lascano, C. E. y E. Palacios, 1993. Intake and digestibility by sheep of mature grass alone and in combinations with two tropical legumes. Tropical Agriculture (Trinidad) 70(4):356-358.

Lascano, C. E. y P. Ávila, 1991. Potencial de producción de leche en pasturas solas y asociadas con leguminosas adaptadas a suelos ácidos. Pasturas Tropicales 13(3):2-10.

Minson, D., 1990. Composición química y valor nutritivo de las leguminosas tropicales. In: Leguminosas Forrajeras Tropicales. FAO. p. 211-219.

Poppi, D. P. y S. R. McLennan, 1995. Protein and energy utilization by ruminants at pasture. J. Animal Sci. 73:278-290. 
Rivera, E. L., 2003. Determinación de digestibilidad y consumo de materia seca de heno de Arachis glabrata en rumiantes. Tesis MS. Universidad de Puerto Rico, Recinto Universitario de Mayagüez.

Rodríguez, A. A., H. L. Díaz, C. Torres y L. Rivera, 2006. Consumo y digestibilidad de nutrientes de heno de maní rizoma perenne y alfalfa comercial. J. Agric. Univ. P.R. 90(3-4):249-251.

Rodríguez, A. A., E. O. Riquelme y P. F. Randel, 1998. Inclusión de leguminosas forrajeras en dietas basadas en gramíneas tropicales. II. Consumo voluntario y digestibilidad aparente de nutrimentos. J. Agric. Univ. P.R. 82(1-2):39-49.

Ruiz, R. y C. M. Vázquez, 1983. Consumo voluntario de pastos tropicales, In: Los Pastos en Cuba. Tomo 2, Utilización. Instituto de Ciencia Animal, La Habana, Cuba.

SAS Institute, 2006. SAS User's Guide, release 9.1 for Windows. SAS Institute, Inc. Cary, North Carolina.

Van Soest, P. J., J. B. Robertson y B. A. Lewis, 1991. Methods for dietary fiber, neutral detergent fiber, and non-starch polysaccharides in relation to animal nutrition. $J$. Dairy Sci. 74:3583-3597.

Villanueva, A. J. F., J. A. B. Cárdenas., J. V. R. Ceja y J. J. B. Guerrero, 2004. Agrotecnia y utilización de Clitoria ternatea en sistemas de producción de carne y leche. Revista Técnica Pecuaria México 42(1):79-96.

Wekesa, F. W., S. A. Abdulrazak y E. A. Mukisira, 2006. The effect of supplementing rhodes grass hay with cotton seed cake and pyrethrum marc based rations on the performance of Sahiwal female weaners. Livestock Research for Rural Development 18(1):1-8. 